\title{
Classification of Normal and Myopathy EMG Signals using BP Neural Network
}

\author{
Mukesh Patidar \\ PG scholar \\ Department of ECE \\ MIT, Mandsaur
}

\author{
Nitin Jain \\ Lecturer \\ Department of ECE \\ MIT, Mandsaur
}

\author{
Ashish Parikh \\ Lecturer \\ Department of ECE \\ MIT, Mandsaur
}

\begin{abstract}
Electromyography (EMG) signal is the muscle electrical activity. Electromyography is a technique for detecting and recording the electrical potential generated by muscle cells. This EMG signals are used in medical professionals to determine specific disorders. This paper basically deals with the analysis of different electromyography signals (NOR \& MYO). In this paper, new method for classification of myopathy patient's and healthy subjects with the help of EMG signal by using back propagation neural network classifier are proposed. This methodology provided $96.75 \%$ accuracy in classification of Myopathy and normal EMG signals.
\end{abstract}

\section{Keywords}

Electromyography, Backpropagation neural network, Myopathy.

\section{INTRODUCTION}

Myopathy is a muscular disease in which the muscle fibers do not function for any one of many reasons, resulting in muscular weakness. Myopathy simply means muscle disease. Electromyography is the detection of the electrical activity associated with muscle contraction. An electromyography (EMG) signal is obtained by measurement of the electrical activity of a muscle during contraction, and reflects the electrical depolarization of excitable muscle fiber membranes that create electrical signals called muscle fibers potentials (MFPs).

Muscle is responsible for producing movements in the body. Every action of the body, voluntary or involuntary, requires muscular motion. Muscle creates the heat, which keeps the body warm and keeps the blood flowing. The ability of the muscles to contract, relax, develop tension and achieve several actions are made possible through various properties of muscles namely extensibility, elasticity, irritability.

The study of electromyography (EMG) signals is a study of the electrical properties and activities of muscle tissue. EMG signals are detected by placing an electrode into, or over a muscle and detecting the extracellular voltages produced by the electrical activity of the muscle fibers. The analysis of EMG signals detected during muscle contraction provides important information to aid in the diagnosis and characterization of neuromuscular disorders [1].

Various features were found in the literatures. For example, features based on time domain are Mean Absolute Value, Modified Mean Absolute Value, Root Mean Square, Integrated of EMG, Simple Square integral, Variance [2][3]. The brachial biceps muscles where used in this study because they were the most frequently investigated in the two abnormal patients.

The non-linear properties may be estimated by calculating nonlinear measures such as entropies, correlation and fractal dimensions, and self-correlation. With the emerging need for identifying complex and subtle actions and gestures, nonlinear methods are becoming more useful in characterizing EMG. Some new approaches in nonlinear methods have been reported [4] for characterization of EMG. These include spectral distribution i.e. Logarithmic representation of EMG spectrum, Poisson representation of EMG spectrum and the method that examines the 'complexity' of raw EMG i.e., Fractal dimension of EMG. Out of these approaches, fractal dimension of EMG has been found sensitive to magnitude and rate of muscle force generated [4]. Gitter et al [5] demonstrated that the fractal characteristics of EMG signal with a dimension is highly correlated with muscle force. Gupta et al [6] reported that the fractal dimension can be used to characterize the EMG signal. Hu et al [7] distinguished two different patterns of fractal dimensions of sEMG signals. These studies demonstrate that fractal information of sEMG is useful for characterizing the signal and identify in properties of the signal. From the literature it is also evident that there are no EMG models that are precise and model the non-linear chaotic properties of muscle activity.

\section{DATA ACQUISITION}

The EMG signal of a normal person and a myopathic patient were collected from the department of neuropathy of Beth Israel Deaconess Medical Center/Harvard Medical School with a Medelec ${ }^{\mathrm{TM}}$ Synergy N2 EMG Monitoring System [Oxford Instruments Medical, Old Working, United Kingdom] which readily provides rapid quantitative EMG analysis. The EMG data were recorded at $50 \mathrm{KHz}$, healthy EMG data and neuropathic EMG data with each sub-set of 1000 samples were considered for this study. The different EMG signals that are taken: 20 normal (NOR) signals and 20 signals suffering from myopathy (MYO).

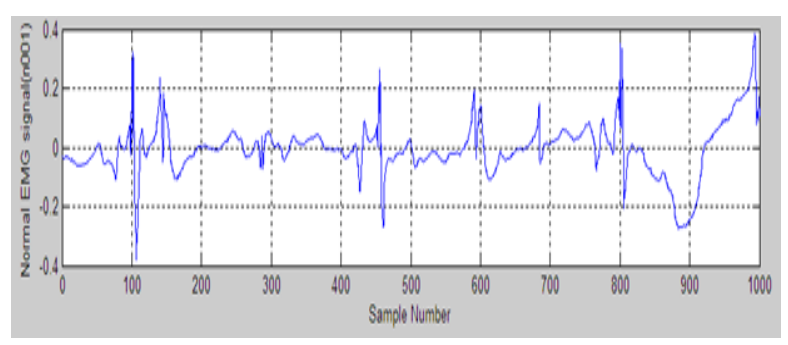

Fig.1 Typical EMG signal for Normal subject 


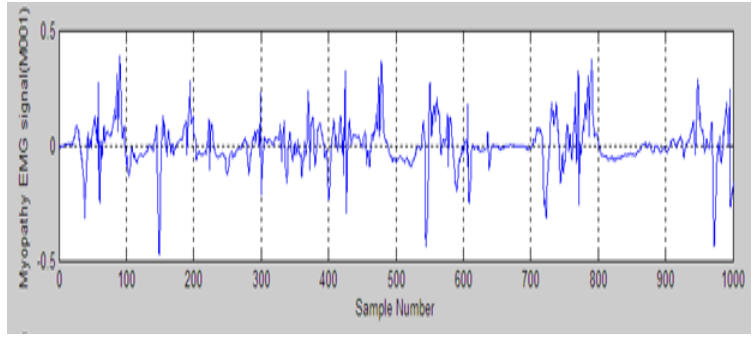

Fig.2 Typical EMG signal for Myopathic subject

\section{METHODOLOGY}

The EMG signal classification involved four step, preprocessing, singular value decomposition, feature extraction and classification using neural network classifier. The block diagram of proposed method shown in the Fig. 3

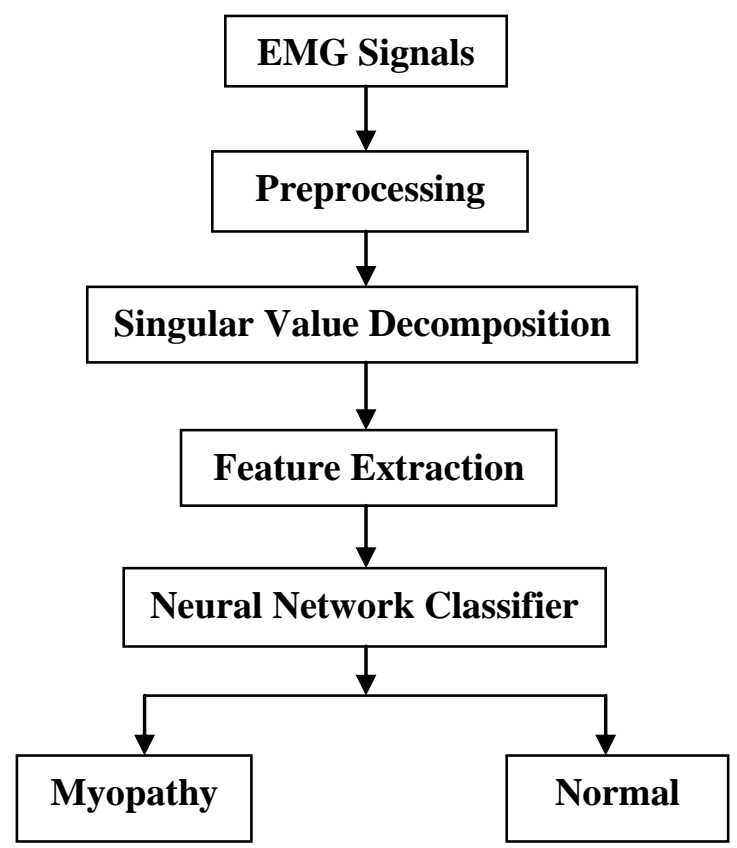

Fig. 3 Block diagram of proposed method

\subsection{Preprocessing}

The preprocessing step the signal $\mathrm{s}[\mathrm{n}]$, where the size of signal is 1000 sample. The EMG signal is non-stationary signal and assume that in 10 sample signal is stationary. So this EMG signal arrange in the matrix of $\mathrm{A}$ is $[10 \times \mathrm{N} / 10]$ by using this step the signal is arrange in to a matrix form [8].

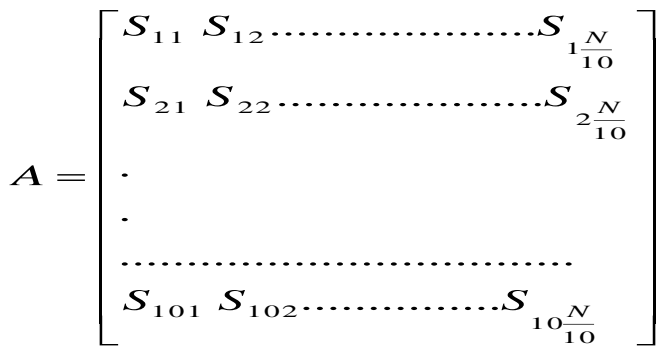

\subsection{Singular value decomposition}

SVD is a matrix factorization technique commonly used for producing low-rank approximations. Given an $\mathrm{m} \times \mathrm{n}$ matrix A, with rank $r$, the singular value decomposition, SVD (A), is defined as

$$
\operatorname{SVD}(\mathrm{A})=\mathrm{U} \times \mathrm{S} \times \mathrm{V}^{\mathrm{T}}
$$

Where $\mathrm{U}, \mathrm{S}$ and $\mathrm{V}$ are of dimensions $\mathrm{m} \times \mathrm{m}, \mathrm{m} \times \mathrm{n}$, and $\mathrm{n} \times \mathrm{n}$, respectively. Matrix $S$ is a diagonal matrix having only $r$ nonzero entries, which makes the effective dimensions of these three matrices $\mathrm{m} \times \mathrm{r}, \mathrm{r} \times \mathrm{r}$, and $\mathrm{r} \times \mathrm{n}$, respectively. $\mathrm{U}$ and $\mathrm{V}$ are two orthogonal matrices and $\mathrm{S}$ is a diagonal matrix, called the singular matrix [9][10].

SVD provides the best low-rank linear approximation of the original matrix A. It is possible to retain only $\mathrm{k}<<\mathrm{r}$ singular values by discarding other entries. Which can be termed as the reduced matrix $S_{k}$. Since the entries in $S$ are sorted i.e., $S_{1} \geq S_{2}$ $\geq \ldots \geq \mathrm{Sr}$, the reduction process is performed by retianing the first $\mathrm{k}$ singular values.

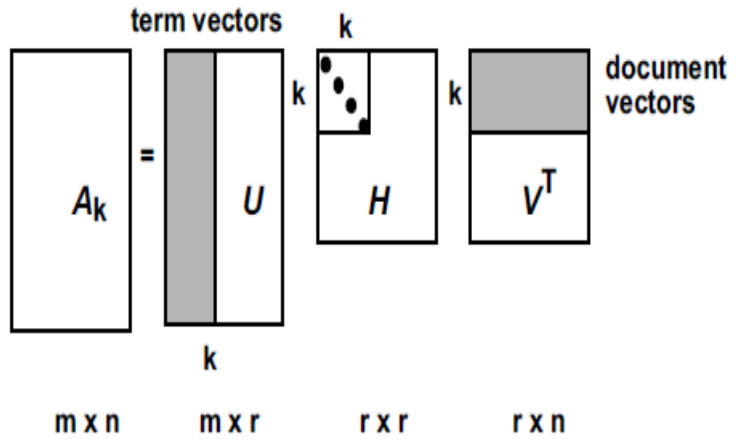

\subsection{Back propagation neural network}

Back propagation neural network is widely used in pattern classification. In theory, a Back Propagation neural network with three layers can solve arbitrary classification. The structure is shown in Fig. 4 [11]. BP neural network is a teacher training network that provides a learning set that consists of some input samples and the known-correct output for each case.

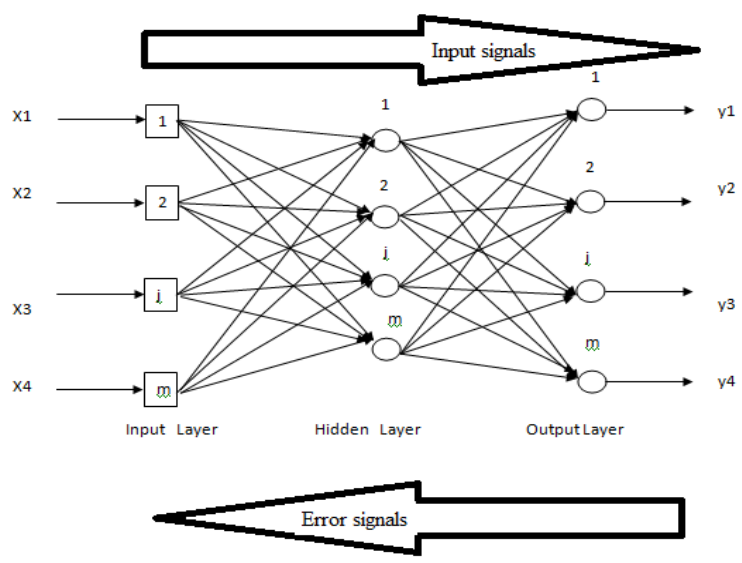

Fig. 4 Back propagation neural network

The network's learning process consist of forward propagation and back propagation. In the forward process, input signals from input layer pass hidden layer and transmit to the output layer. If there is a difference which is defined as error value between the actual output values and knowncorrect output values, then BP network turns to the process of error back propagation [12] 


\section{RESULTS AND DISCUSSION}

In this study, the EMG signal is represent in the matrix. The matrix of the signal is $10 * 100$ used to find the 10 singular vector by decomposed matrix in to help of singular value decomposition. The singular vector used for classification of normal and myopathy EMG signal by using back propagation neural network.

In this study, performance evaluation parameters namely sensitivity, specificity, and accuracy were used. Sensitivity is the probability that the test gives a positive result when abnormal features (i.e., features corresponding to myopathic patients) are tested. The specificity of a test is the probability that a test gives a negative result when normal cases are tested. Accuracy is the ratio of correctly classified samples to the total number of samples used.

The whole dataset was divided into two parts. One part used for training $(60 \%)$ and other part used for testing $(40 \%)$. The $\mathrm{n}$ during the first fold, two parts of the data were used as training data for developing the classifier. The remaining part of the data was used as the test data, and the evaluation parameters, namely, Accuracy, Sensitivity, and Specificity were calculated. This procedure was repeated ten times by using a different test set in each fold. The averages of the performance measures obtained in each fold were reported as the final performance measures.

Specificity: number of correct classified healthy subjects/number of total healthy subjects.

Sensitivity (myopathy): number of correct classified subjects suffering from myopathy/number of total subjects suffering from myopathy.

Total classification accuracy: number of correct classified subjects/number of total subjects [13].

$$
\begin{gathered}
\operatorname{SEN}(\%)=\frac{T P}{T P+F N} \times 100 \\
\operatorname{SPE}(\%)=\frac{T N}{T N+F P} \times 100 \\
A C C(\%)=\frac{T P+T N}{T P+T N+F P+F N} \times 100
\end{gathered}
$$

Where TP is the true positive, the total number of correctly detected positive events; $\mathrm{TN}$ is the true negative, the total number of correctly detected negative events; FP is the false positive, the total number of erroneously positive detection; and $\mathrm{FN}$ is the false negative, the total number of erroneously negative detection.

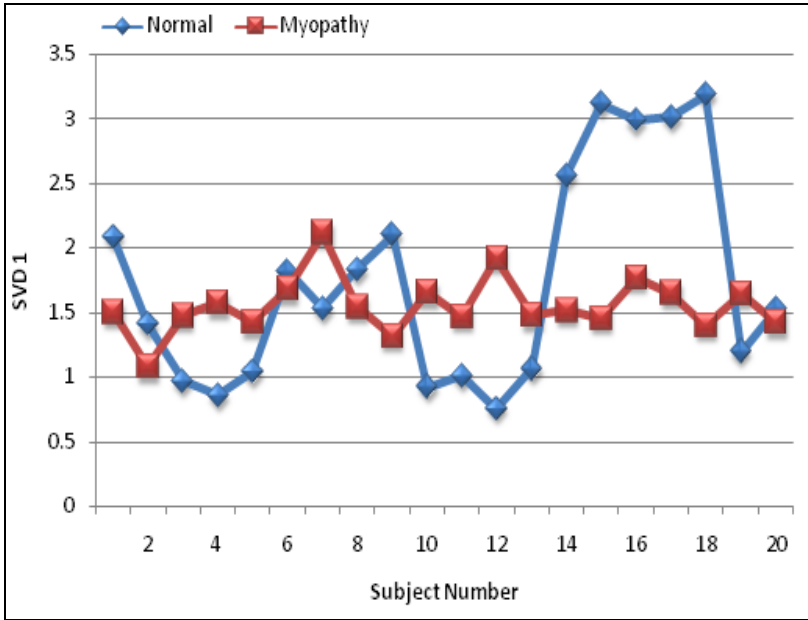

Fig. 5 Comparison of Normal and Myopathy SVD1

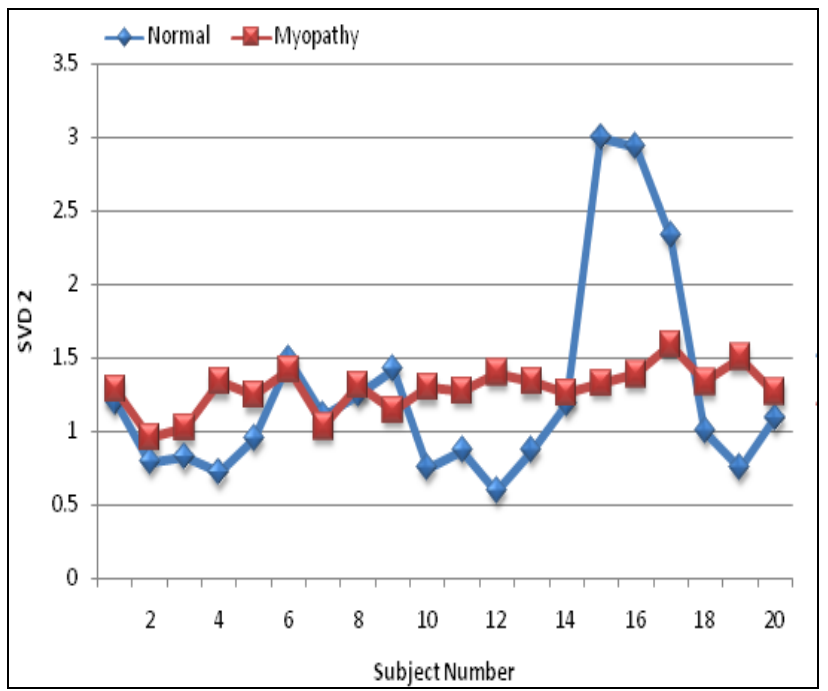

Fig. 6 Comparison of Normal and Myopathy SVD2

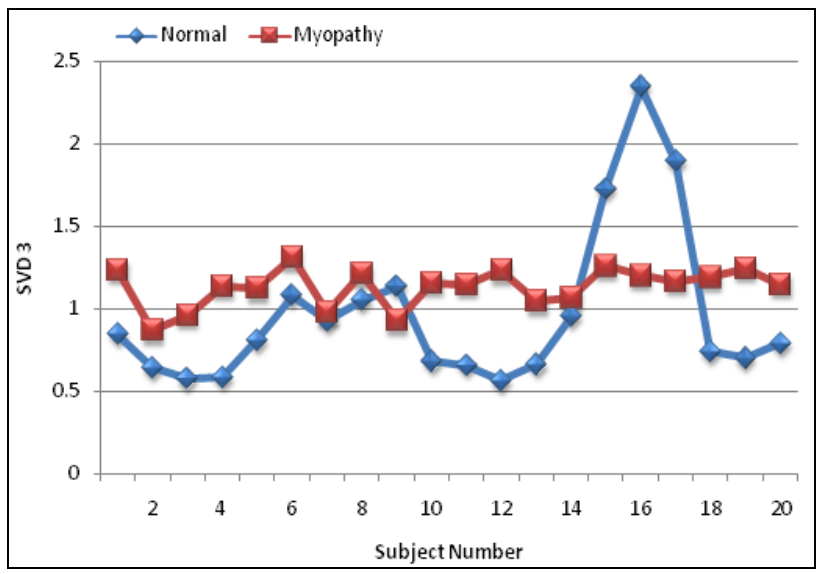

Fig. 7 Comparison of Normal and Myopathy SVD3 


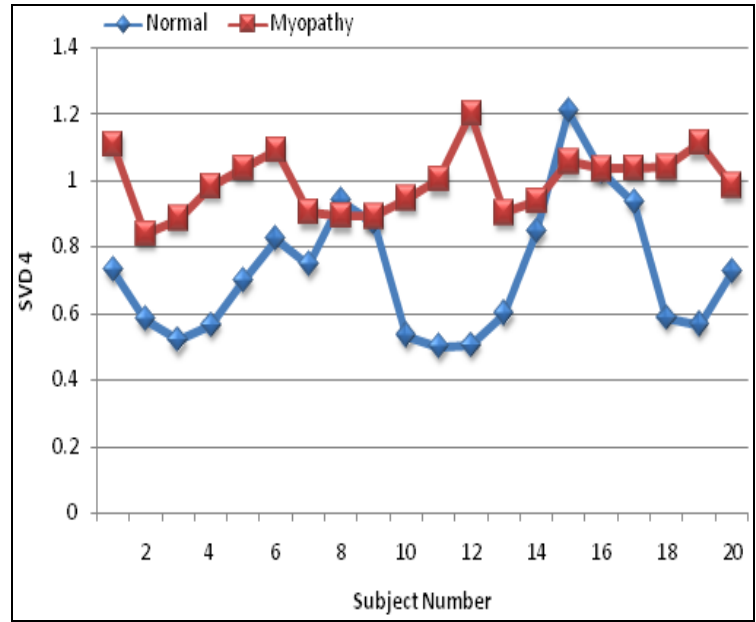

Fig. 8 Comparison of Normal and Myopathy SVD4

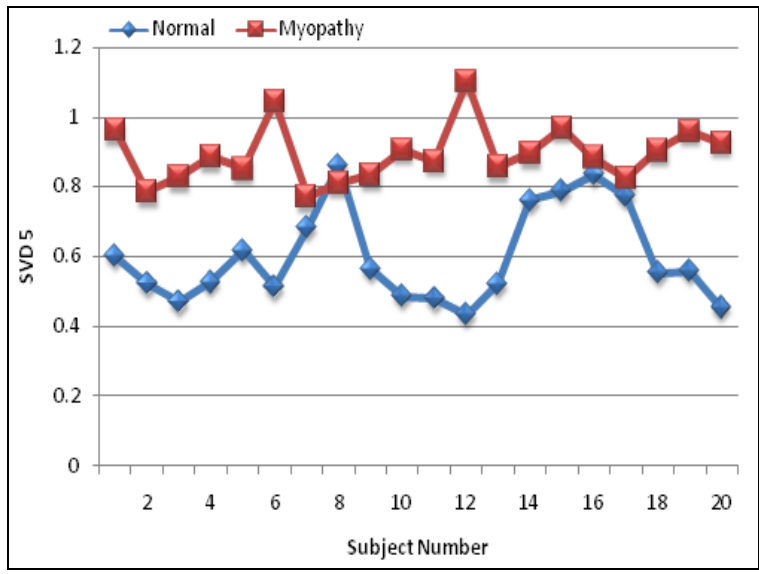

Fig. 9 Comparison of Normal and Myopathy SVD5

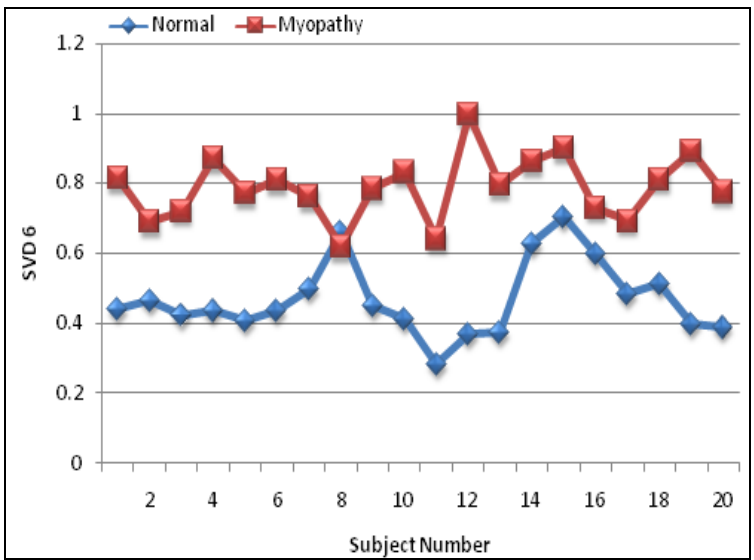

Fig. 10 Comparison of Normal and Myopathy SVD6

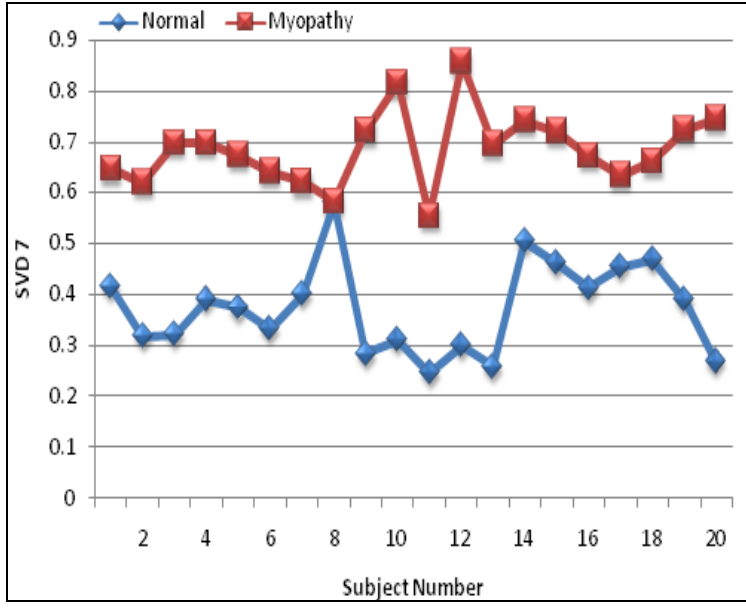

Fig. 11 Comparison of Normal and Myopathy SVD7

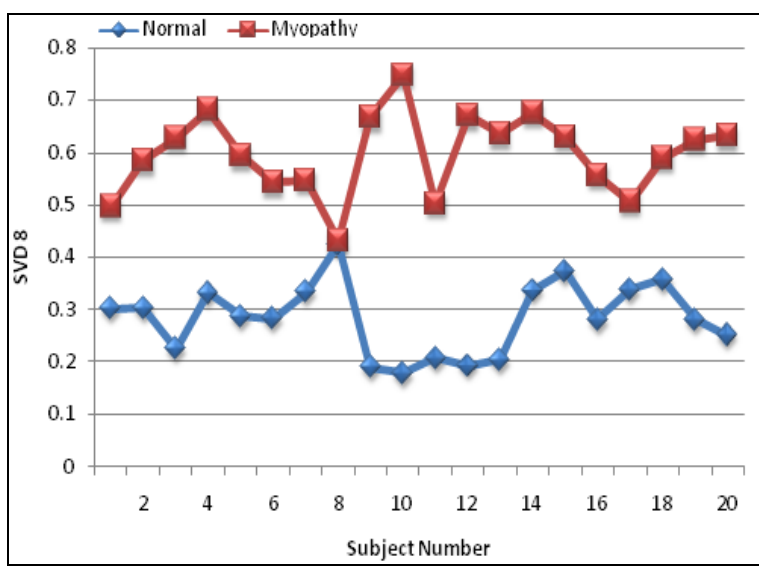

Fig. 12 Comparison of Normal and Myopathy SVD8

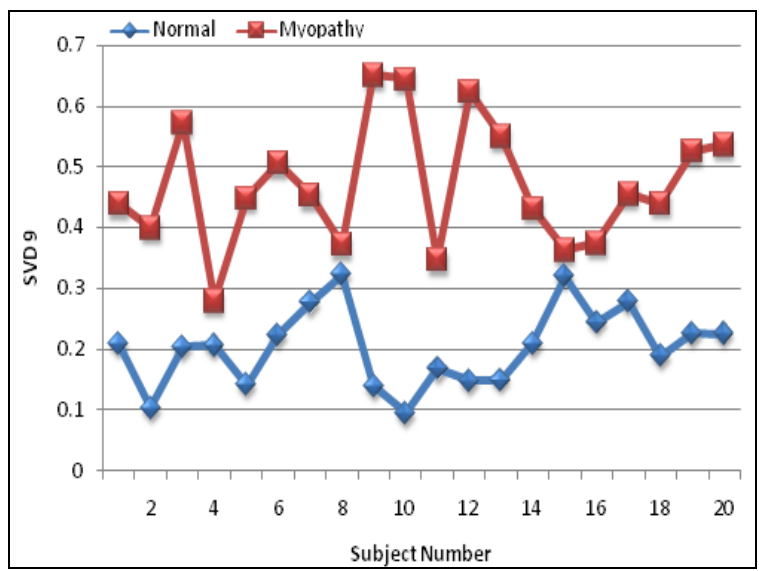

Fig. 13 Comparison of Normal and Myopathy SVD9 


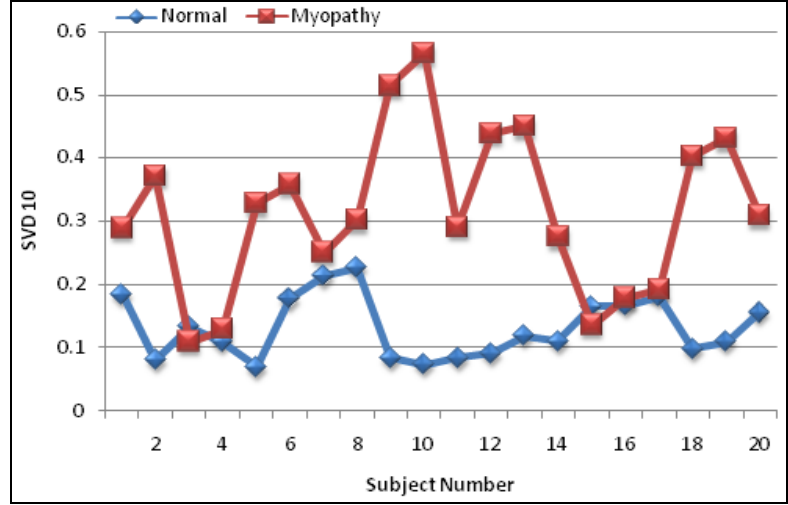

Fig. 14 Comparison of Normal and Myopathy SVD10

TABLE 1: Sensitivity, Specificity and Accuracy values

\begin{tabular}{|c|c|c|c|c|c|c|c|}
\hline Trial & $\mathrm{TP}$ & $\mathrm{TN}$ & FP & FN & $\begin{array}{l}\text { SEN } \\
(\%)\end{array}$ & $\begin{array}{l}\text { SPE } \\
(\%)\end{array}$ & $\begin{array}{l}\text { ACC } \\
(\%)\end{array}$ \\
\hline 1 & 20 & 20 & 0 & 0 & 100 & 100 & 100 \\
\hline 2 & 17 & 20 & 3 & 0 & 100 & 86.95 & 92.5 \\
\hline 3 & 19 & 20 & 1 & 0 & 100 & 95.23 & 97.5 \\
\hline 4 & 20 & 19 & 0 & 1 & 95.23 & 100 & 97.5 \\
\hline 5 & 20 & 16 & 0 & 4 & 83.33 & 100 & 90 \\
\hline 6 & 19 & 20 & 1 & 0 & 100 & 95.23 & 97.5 \\
\hline 7 & 20 & 20 & 0 & 0 & 100 & 100 & 100 \\
\hline 8 & 20 & 18 & 0 & 2 & 90.90 & 100 & 95 \\
\hline 9 & 19 & 20 & 1 & 0 & 100 & 95.23 & 97.5 \\
\hline 10 & 20 & 20 & 0 & 0 & 100 & 100 & 100 \\
\hline \multicolumn{5}{|c|}{ Average } & 96.94 & 97.26 & 96.75 \\
\hline
\end{tabular}

\section{CONCLUSION AND FUTURE WORK}

The Singular value decomposition process is a useful and powerful method to decompose EMG signal into a set of Different singular value. The singular values extracted from the preprocessed EMG signals have been found useful in classification of Myopathy and normal EMG signals. Finally, it is conclude that the Singular values are effective for classification of Myopathy and normal EMG signals. The classification results indicated that the neural network had provided $96.75 \%$ accuracy in classification of Myopathy and normal EMG signals. Future direction of research may include application of singular value for different psychological states from EMG signals.

\section{REFERENCES}

[1] S.Boisset, F Goubel, "Integrated electromygraphy activity and muscle work," J Applied Physiol, vol 35, pp. 695-702, 1972.

[2] R. Plonsey, "The active fiber in a volume conductor," IEEE Trans Biomed Eng, vol. 21, pp. 371-381, 1974.

[3] Carlo De Luca „Electromyography “.Encyclopedia Of Medical Devices and Instrumentation (John G. Webster, Ed), John Wiley Publisher, 2006.

[4] M. A. Nussbaum, "Localized Muscle Fatigue" in Lecture Notes on Advanced Methods in Occupational Biomechanics,http://www.nussbaum.org.vt.edu/courses.h tm, Last Access: Aug-2006.

[5] J. A. Gitter, and M. J. Czerniecki, "Fractal analysis of electromyographic interference pattern," Journal of neuroscience Methods, vol. 58, pp. 103-108, 1995.

[6] V. Gupta, S. Suryanarayanan, and N. P. Reddy,"Fractal analysis of surface EMG signals from the biceps," Intl. J. Medical informatics vol. 45, pp. 185-192, 1997

[7] X. Hu, Z. Wang, and X .Ren, "Classification of surface EMG signal with Fractal dimension," Journal of Zhejiang University Science, vol. 6, no. 8, pp.844-848, 2005.

[8] G. Golub and C. Van Loan, Matrix computation (2nd Ed.) Baltimore, MD; John Hopkins, 1989.

[9] Kirk Baker "Singular Value Decomposition Tutorial" March 29, 2005 (Revised January 14, 2013).

[10] Trefethen, Lloyd N.; Bau III, David. Numerical linear algebra. Philadelphia: Society for Industrial and Applied Mathematics. 1997 ISBN 978-0-89871-361-9.

[11] Stuart Russell and Peter Norvig. Artificial Intelligence A Modern Approach. p. 578. "The most popular method for learning in multilayer networks is called Backpropagation. It was first invented in 1969 by Bryson and Ho, but was largely ignored until the mid-1980s."

[12] Arthur Earl Bryson, Yu-Chi Ho (1969). Applied optimal control: optimization, estimation, and control. Blaisdell Publishing Company or Xerox College Publishing. pp. 481.

[13] Abdulhamit Subasi, Mustafa Yilmaz, Hasan Riza Ozcalik, "Classification of EMG signals using wavelet neural network", Journal of Neuroscience Methods 156 (2006). pp. 366. 